\title{
Erratum to: Value of adding sonoelastography to conventional ultrasound in patients with congenital muscular torticollis
}

\author{
So-Yeon Lee • Hee-Jin Park • Yoon Jung Choi • \\ Seon Hyeong Choi • Shin Ho Kook • Myong-Ho Rho • \\ Eun Chul Chung
}

Published online: 1 October 2013

(C) Springer-Verlag Berlin Heidelberg 2013

Erratum to: Pediatr Radiol

DOI 10.1007/s00247-013-2750-x

The original version of the paper, unfortunately, contained errors.

Typographical errors were found in Table 5. The correct Table 5 is presented below.

Table 5 US findings of sternocleidomastoid muscle between patients with heterogeneous sternocleidomastoid muscle and the control group, median (interquartile range). The first and second rows in each finding represent the results determined by reviewers 1 and 2 , respectively

\begin{tabular}{|c|c|c|c|}
\hline Finding & $\begin{array}{l}\text { Patients with heterogeneous } \\
\text { sternocleidomastoid muscle }\end{array}$ & Controls & $P$ \\
\hline \multirow[t]{2}{*}{ Thickness of the sternocleidomastoid muscle (mm) } & $12.0(8.7-18.0)$ & $5.3(4.6-6.9)$ & $0.001 *$ \\
\hline & $12.1(8.5-17.4)$ & $5.2(4.8-6.9)$ & $0.001 *$ \\
\hline \multirow[t]{2}{*}{ Difference from thickness of contralateral sternocleidomastoid muscle (mm) } & $5.3(3.5-13.3)$ & $0.0(0.0-0.1)$ & $<0.001^{*}$ \\
\hline & $5.1(3.5-13.0)$ & $0.0(0.0-0.4)$ & $<0.001^{*}$ \\
\hline \multirow{2}{*}{$\begin{array}{l}\text { Ratio (thickness of sternocleidomastoid muscle/thickness of contralateral } \\
\text { sternocleidomastoid muscle) }\end{array}$} & $2.8(1.7-3.8)$ & $1.0(1.0-1.0)$ & $<0.001^{*}$ \\
\hline & $2.6(1.7-3.9)$ & $1.0(1.0-1.1)$ & $0.001 *$ \\
\hline \multirow[t]{2}{*}{ Elastography score } & $3.0(2.5-3.0)$ & $2.0(1.0-2.0)$ & $0.001 *$ \\
\hline & $3.0(2.5-3.0)$ & $2.0(1.0-2.0)$ & $0.001 *$ \\
\hline
\end{tabular}

The online version of the original article can be found at http://dx.doi.org/ 10.1007/s00247-013-2750-x.

S.-Y. Lee $\cdot$ H.-J. Park $(\bowtie) \cdot$ Y. J. Choi $\cdot$ S. H. Choi $\cdot$ S. H. Kook $\cdot$

M.-H. Rho $\cdot$ E. C. Chung

Departments of Radiology, Kangbuk Samsung Hospital,

Sungkyunkwan University School of Medicine, 108 Pyeong-dong,

Jongno-gu, Seoul 110-746, South Korea

e-mail: radiology12@paran.com 